\title{
Factors Influencing Depression among Elderly Patients in Geriatric Hospitals
}

\author{
Young Ju Jee, RN, $\mathrm{PhD}^{1)}$, Yun BoK Lee, RN, $\mathrm{PhD}^{1) *}$ \\ 1) Department of Nursing, College of Natural Science, Kyungnam University: 7 Kyungnamdaehak-ro, \\ Masanhappo-gu, Changwon-si, Gyeongsangnam-do 631-701, Republic of Korea
}

\begin{abstract}
Purpose] The purpose of this study was to investigate the prevalence of depression among elderly patients and identify the factors influencing depression in a geriatric hospital in Korea. [Subjects] A self-report questionnaire was administered to the patients in community geriatric hospitals. Participants were 195 elderly patients. [Methods] The instruments utilized in this study were the Geriatric Depression Scale Short Form Korea (GDSSF-K), an activity of daily living scale, a self-esteem scale, a social support scale, and a life satisfaction scale. Data were collected from April 20 to June 20, 2011. Data were analyzed using descriptive statistics, Pearson correlations, and multiple logistic regression analysis using SPSS 15.0 and Stata 11.0. [Results] The average GDSSF-K score of the participants was 8.94 , indicating a moderate level of depression. About $78.4 \%$ of the elderly patients in this study were classified as predisposed toward depression. Significant predictors for depression included the patients' perceived health status and life satisfaction. [Conclusion] The results indicate that elderly patients face a high risk of developing depression and that efforts should be made to address it wherever possible. Regular depression screening will be beneficial for early detection of depression in patients at community geriatric hospitals.

Key words: Depression, Elderly, Nursing care
\end{abstract}

(This article was submitted Apr. 23, 2013, and was accepted Jun. 20, 2013)

\section{INTRODUCTION}

Depression is becoming a very important issue in modern society. With ensuing social problems to be expected, it may become the disease with the greatest social burden after ischemic heart disease ${ }^{1,2)}$. Considering that depression is the most common psychological problem in old age ${ }^{3)}$, its management in the elderly population is essential.

More psychological problems will develop in older adults residing in rehabilitation clinics as the number of visitors they receives declines with time. Depression will be one of the most common problems in old age. Suicide ideation or recurring thoughts of death are common in people suffering from depression. Elderly people with depression have a particularly poor prognosis as compared with people in other age groups, as they have a relatively higher risk of suicide and mortality $\left.{ }^{4}, 5\right)$. Factors correlating with depression include reduced ability in daily activities ${ }^{6}$, reduced level of self-respect, reduced satisfaction with life, pain $^{7-10)}$, lower economic standards and income ${ }^{11)}$, fatigue ${ }^{12)}$, and absence of social support ${ }^{13}{ }^{14}$ ). Previous studies on depression in elderly patients include studies that have compared selfreported depression between those who live in hospitals and

*Corresponding author. Yun Bok Lee, (e-mail: caggg@ kyungnam.ac.kr)

(C2013 The Society of Physical Therapy Science

This is an open-access article distributed under the terms of the Creative Commons Attribution Non-Commercial No Derivatives (by-ncnd) License $<$ http://creativecommons.org/licenses/by-nc-nd/3.0/> . at home ${ }^{15)}$ and the relationship between depression and ill health in elderly patients in rehabilitation clinics ${ }^{16}$. However, these studies either used simplistic correlation between depression and a couple of variables of interest or studied depression as a scale on a continuous variable. Accordingly, they have been restricted to research on the linear correlation of depression with related factors and patients' severity of depression. Few studies have categorized their sample clinically into different groups of depression, or used scales with cut-offs that are indicative of categories of clinical diagnoses.

Against this background, the purpose of this study was to highlight the prevalence of depression in elderly patients residing in rehabilitation clinics, determine potential predictors of depression in them, and inform the development of preventive programs with our results.

\section{SUBJECTS AND METHODS}

This was a cross-sectional study that explored the prevalence of depression and its predictors in a group of 195 elderly patients from four geriatric hospitals in South Korea. Patients who have been hospitalized for more than three months comprised the target group for this study, as this is known to cause severe anxiety and depression due to the change in redidence ${ }^{17}$. The main researcher approached these patients to communicate the details of participation, which included the study's purpose, confidentiality of response and research data, and freedom of withdrawal. The inclusion criteria were (1) no psychological diseases, (2) a Korean Mini-Mental State Examination (K-MMSE) score 
higher than $18^{18)}$, (3) residence at the rehabilitation clinic for more than 3 months, and (4) having an understanding of the purpose of the study and willingness to participate. Sample characteristics are shown in Table 1. Power analysis revealed that the current sample size was sufficient for obtaining a medium effect size, which is considered acceptable in most studies ${ }^{2}$. It also exceeded the minimum sample size of 115 required for multiple logistic regression analysis. $G^{*}$ power 3.1 was used to calculate the sample size required to produce a medium effect size with 11 predicted variables at $\alpha=0.05$ and $\beta=0.20$. Data collection was carried out by four research assistants, trained by the researcher, in the period of April 20 to June 20, 2011. Approval was obtained from the four geriatric hospitals, and consent was obtained from the patients who agreed to participate. There is no institutional review board overseeing studies being carried out within small organizations such as the four rehabilitation clinics approached in this study. Therefore, permission was obtained from the guardians of the patients on a "Patient's Family Visiting Day" after explaining the study's purpose and methods. The questionnaire was supposed to be answered by the participants. However, the researcher or research assistants read the questionnaire to patients who required help and filled in answers on such patients' behalf. Among the 200 questionnaires distributed, all were returned (response rate: 100\%), and 5 questionnaires with incomplete response were excluded.

In measuring depression, the questions used consisted of the scale developed by Sheikh and Yesavage ${ }^{19)}$, and 15 questions from the Geriatric Depression Scale Short Form Korean Version as modified by $\mathrm{Ki}^{19}$ ). Each item was coded as "1" for "Yes" and "0" for "No," while questions 1, 3, 4, 5, $6,9,10,13,14$, and 15 were reverse coded. A higher score indicated greater severity of depression, and based on the studies by Sheikh and Yesavage ${ }^{19)}$, and $\mathrm{Ki}^{20)}$, a cut-off point of 5 was used. A score of more than including 5 was considered to indicate being depressed, and a score of less than 5 was considered to indicate not depressed (nondepressed). Reliability analyses revealed that the scales used in Ki's study $^{20)}$ and the current study achieved Cronbach's $\alpha$ coefficients of 0.88 and 0.85 respectively.

In measuring the ability to perform daily activities, the 8 questions used were items regarding activities of daily living (ADL), which was developed by Song ${ }^{21}$. Items were scored on a 4-point Likert scale in which "1" represents "possible" and "4" represents "very well." A higher score represented greater ability to perform daily activities. At the time of development, the Cronbach's $\alpha$ of Song's instrument ${ }^{21)}$ was 0.98 , and its Cronbach's $\alpha$ was 0.91 in this study.

To measure self-esteem, the 10-question tool developed by Rosenberg was used ${ }^{22}$. Each item was scored on a 5-point Likert scale with options varying from " 1 " for "almost never" to "5" for "almost always." Questions 5, 7, 8,9 , and 10 were reverse coded. A higher score indicated greater self-esteem. The translated version of the scale has a Cronbach's $\alpha$ of 0.63 in the study by Kim and Kang ${ }^{7)}$ and a Cronbach's $\alpha$ of 0.80 in the current study.

To assess social support, the scale modified by Jang was used ${ }^{23}$. This scale was based on the social support
Table 1. General characteristics of the subjects

\begin{tabular}{|c|c|c|c|}
\hline Characteristics & Classification & $\mathrm{n}$ & $\%$ \\
\hline \multirow{2}{*}{ Gender } & Male & 49 & 25.1 \\
\hline & Female & 146 & 74.9 \\
\hline \multirow{3}{*}{$\begin{array}{l}\text { Age } \\
\text { (years) }\end{array}$} & $65-69$ & 24 & 12.3 \\
\hline & $70-79$ & 89 & 45.6 \\
\hline & $80-89$ & 82 & 42.1 \\
\hline \multirow{4}{*}{ Education } & None & 74 & 37.9 \\
\hline & Elementary school & 69 & 35.4 \\
\hline & Middle school & 21 & 10.8 \\
\hline & High school or above & 31 & 15.9 \\
\hline \multirow{4}{*}{ Religion } & None & 50 & 25.6 \\
\hline & Buddhist & 84 & 43.1 \\
\hline & Christian & 51 & 26.2 \\
\hline & Catholic and others & 10 & 5.1 \\
\hline \multirow{2}{*}{ Spouse } & None & 161 & 82.6 \\
\hline & Have & 34 & 17.4 \\
\hline \multirow{2}{*}{ Children } & Have & 181 & 92.8 \\
\hline & None & 14 & 7.2 \\
\hline \multirow{3}{*}{$\begin{array}{l}\text { Economic } \\
\text { status }\end{array}$} & High & 12 & 6.2 \\
\hline & Middle & 126 & 64.6 \\
\hline & Low & 57 & 29.2 \\
\hline \multirow{3}{*}{$\begin{array}{l}\text { Perceived } \\
\text { health status }\end{array}$} & $\mathrm{Bad}$ & 138 & 70.8 \\
\hline & Moderate & 37 & 19.0 \\
\hline & Good & 20 & 10.3 \\
\hline
\end{tabular}

Values are numbers

index developed by Park ${ }^{24)}$. The aspects of support were categorized into support provided by hospital staff, volunteers, colleagues, relatives, and non-relatives. There were 30 questions within each category of support. Items were measured against a 3-point Likert scale that ranged from "1" for "never" to " 3 " for "always." A higher score represented higher levels of social support. The Cronbach's $\alpha$ of the scale in Jang' ${ }^{23)}$ study was 0.81 , and that in the current study $^{24)}$ is 0.84 .

In measuring satisfaction with life, the 20-question tool developed by Choi was used ${ }^{25)}$. The items measured past, present, and future satisfaction with life from various aspects including psychological and environmental points of view. Each question was answered on a 3-point Likert scale with " 1 " representing "no" and " 3 " representing "yes." A higher score represented a greater satisfaction with life. At the time of development of this index, the Cronbach's $\alpha$ was 0.83 , and Cronbach's $\alpha$ was 0.62 in this study.

Data collected were analyzed using the Statistical Package for the Social Sciences (SPSS) 15.0 and Stata 11.0. $\chi^{2}$ tests and t-tests were conducted to compare the depressed patients with the nondepressed patients with regard to various variables of interest. In examining the predictive factors of depression, multiple logistic regression analysis was performed using the forward selection method. Factors found to be significant at $\mathrm{p}<0.05$ in the univariate analyses were set as the independent variables, and the level of depression was set as the dependent variable. The limits of entry 
Table 2. Comparison of variables between the nondepressed and depressed groups

\begin{tabular}{lccc}
\hline \multirow{2}{*}{ Variables } & $\begin{array}{c}\text { Categories } \\
(\text { mean })\end{array}$ & $\begin{array}{c}\text { Nondepressed group } \\
(\mathrm{n}=42)\end{array}$ & $\begin{array}{c}\text { Depressed group } \\
(\mathrm{n}=153)\end{array}$ \\
\cline { 3 - 4 } & & Mean \pm SD or $\mathrm{n}(\%)$ & Mean \pm SD or n (\%) \\
\hline GDSSF-K & $8.94 \pm 1.53$ & $3.45 \pm 1.53$ & $10.45 \pm 1.53$ \\
Gender & Male & $16(8.2)$ & $33(16.9)$ \\
& Female & $26(13.3)$ & $120(61.5)$ \\
Age (years) & $65-69$ & $6(3.1)$ & $18(9.2)$ \\
& $70-79$ & $16(8.2)$ & $73(37.4)$ \\
& $80-89$ & $20(10.3)$ & $62(31.8)$ \\
Education & None & $12(6.2)$ & $62(31.8)$ \\
& Elementary school & $14(7.2)$ & $55(28.2)$ \\
Perceived health status & Middle school & $7(3.6)$ & $14(7.2)$ \\
& High school or over & $9(4.6)$ & $22(11.3)$ \\
& Bad & $21(10.8)$ & $117(60.0)$ \\
Activity of daily living & Moderate & $11(5.6)$ & $26(13.3)$ \\
Social support & Good & $10(5.1)$ & $10(5.1)$ \\
Self-esteem & & $37.79 \pm 10.54$ & $31.43 \pm 9.15$ \\
Life satisfaction & & $44.74 \pm 8.00$ & $43.35 \pm 8.55$ \\
\hline
\end{tabular}

Values are means $\pm \mathrm{SD}$

and removal of the independent variables were set at 0.05 and 0.10 , respectively, and a cross ratio and $95 \%$ confidence interval were given for each factor. The model's predictive power for depression was analyzed using the receiver operating characteristic (ROC) curve and by evaluating the model's accuracy of categorization.

\section{RESULTS}

Gender $\left(\chi^{2}=4.78, p=0.026\right)$, state of health $\left(\chi^{2}=12.73\right.$, $\mathrm{p}=0.002)$, ability to perform everyday activities $(t=-3.86$, $\mathrm{p}<0.001)$, level of self-esteem $(t=-3.80, \mathrm{p}<0.001)$, and satisfaction with life $(t=-7.93, \mathrm{p}<0.001)$ significantly differed between depressed and nondepressed subjects (Table 2). Of those who were depressed, $61.5 \%$ were female. Participants who were depressed accounted for less than $10 \%$ of those who were in their $60 \mathrm{~s}$ but $69.2 \%$ of those in their $70 \mathrm{~s}$. There were no significant differences between depressed and nondepressed participants in terms of age $\left(\chi^{2}=7.05, p=0.133\right)$, education level $\left(\chi^{2}=3.90, p=0.272\right)$, and perceived level of social support $(t=-0.95, \mathrm{p}=0.345)$.

To examine the predictors of depression, factors that were significantly different between the depressed and nondepressed were included within the logistic regression. Predictability testing for these factors using simple logistic regression showed that all of them were significant $(p<0.05)$, and hence, all five of these variables were included within the regression formula. Using the forward selection method, multiple logistic regression analysis revealed that state of health $(p=0.030)$ and satisfaction with life $(p<0.001)$ emerged as significant. A Hosmer-Lemeshow test of the regression model suggested that there was an acceptable fit between the model's estimate and the data $\left(\chi^{2}=7.14\right.$, degrees of freedom $(d f)=8, \mathrm{p}=0.521)$. The null hypothesis, which stated that the model with two predictors and the basic model with only intercepts (constant) in the entire model coefficient test were the same, was rejected. This was because the former model was significant following the Hosmer-Lemeshow test $\left(\chi^{2}=65.55, d f=5, \mathrm{p}<0.001\right)$. The model with two predictors was evaluated as having better fit than the basic model. In the regression model, the dependent variable explained 44\% (Nagelkerke's $R^{2}$ ) of the variance. The percentage of the depression group status explained for $95.4 \%$ of the dependent variable. In terms of odds ratios, the likelihood of depression decreased by 2.02 times with every unit increase in the patient's state of health, and the likelihood of depression increased by 1.47 times with every unit increase in a patient's satisfaction with life. In other words, the likelihood of depression was expected to increase with a lower state of health as well as satisfaction with life.

\section{DISCUSSION}

The purpose of this study was to identify the prevalence of depression in a group of elderly patients from rehabilitation clinics and the difference in characteristics between the depressed and nondepressed groups. Further, this study aimed to highlight significant predictors of depression and hence inform the development of preventive programs.

The present study found that $78.4 \%$ of the elderly patients were depressed, and this is higher than the $45.1 \%$ of elderly patients found to be depressed in general hospitals in a previous study by $\mathrm{Shin}^{26)}$. However, this difference can be explained by the lower cut-off point of 5 used in this study as compared with the original cut-off point of 8 used in Shin's study. Since the present study and Sheikh and Yesavage's ${ }^{19)}$ 
study used the same cut-off point of 5, comparison with this latter study would be better. Further, the Korean patients in Shin's ${ }^{26)}$ study scored a mean depression score of 7.

The present results revealed no differences between the depressed and nondepressed groups based on age, education level, and perceived social support but revealed significant differences in gender, state of health, ability to perform daily activities, level of self-respect, and satisfaction with life. These results, while similar to those from a study by Yoo and $\mathrm{Lim}^{16)}$, were different from those of the study of Lee and Lee ${ }^{27)}$ study, in which age was significantly different between the study groups. The difference in context of these studies was that while the current study and that by Yoo and $\mathrm{Lim}^{16)}$ recruited hospitalized elderly patients from clinics and hospitals, the participants in the study of Lee and Lee ${ }^{27)}$ were elderly patients in long-term rehabilitative care residing in the community. For the elderly patients who were hospitalized, age was not significantly different between the depressed and nondepressed groups, perhaps due to an overriding health problem they were facing. However, there might be a relationship between age and depression for older adults who are living independently and are generally in good health. In general, there is a stigma towards growing old that might lead to an increase in depression with age. As such, it is necessary to change the aging culture to one where people are very comfortable in accepting the natural course of aging. This can be done by portraying a positive image of aging through education. In the study by Seo, Jung, and $\mathrm{Kim}^{28)}$, education and social support were not significantly related to depression. The level of social support of these participants was below average regardless of their depression status, and this was likely due to feeling detached from their family members after a long period of hospitalization. However, Seo, Jung, and $\mathrm{Kim}^{28)}$ found that for older adults who lived at home, level of satisfaction towards their family members was not significantly related to depression. Therefore, although depression in the elderly is affected by close friends and family members for older adults living at home and in hospitals, this factor does not yield any significant difference. The current study revealed that gender, state of health, ability to perform daily activities, level of self-respect, and satisfaction with life are significantly related to depression. The conclusion of this study is similar to that in the study of Kang and Jung ${ }^{29)}$, who stated that the level of depression in elderly Koreans is more affected by psychological and physical factors than social and demographical factors. Psychological factors include satisfaction with life and level of self-esteem, and physical health includes level of comorbidity and ability to perform daily activities. Social and demographical factors include gender, age, education level, and religion. One difference between the studies is that while Kang and Jung's sample had similar numbers of men and women (93 men and 108 women), the current sample had a larger proportion of women (49 men and 108 women). While a number of factors affected depression, state of health and satisfaction with life were significant predictors of depression.

The present results suggest that health-care providers in rehabilitation clinics can have the biggest influence on pa- tients' psychological well-being, especially in terms of being a source of emotional support. A multilevel approach is recommended, and this includes educational mediation programs, volunteer services, health enhancement programs, and physical programs for the elderly.

Limitations of this study include its cross-sectional nature and the limited population from which the sample was obtained. Its findings should be generalized with caution.

\section{ACKNOWLEDGEMENT}

This work was supported by Kyungnam University Research Fund, 2013.

\section{REFERENCES}

1) Murray CL, Lopez AD: The global burden of disease. MA: Harvard University Press, 1996.

2) Polit DF, Sherman RE: Statistical power in nursing research. Nurs Res, 1990, 39: 365-369. [Medline] [CrossRef]

3) Stek ML, Vinkes DJ, Gussekloo J, et al.: Natural history of depression in the oldest old: population based prospective study. Br J Psychiatry, 2006, 188: 65-69. [Medline] [CrossRef]

4) Heun R, Hein S: Risk factors of major depression in the elderly. Eur Psychiatry, 2005, 20: 199-204. [Medline] [CrossRef]

5) Cole MG, Dendukuri N: Risk factors for depression among elderly community subject: a systematic review and meta analysis. Am J Psychiatry, 2003, 160: 1147-1156. [Medline] [CrossRef]

6) Ann KS: Study on the quality of life and social support of married couples and single households elderly. J Korean Geriatr Soc, 2005, 25: 1-19.

7) Kim HK, Kang KJ: A study of correlation between self- esteem and selfcare agency of elderly inpatients. J Korean Gerontol Nurs, 2000, 2: 7-21.

8) Hur JS, Yoo SH: Determinants of depression among elderly persons. Ment Health Soc Work, 2002, 13: 7-35.

9) Kim YH: Factors discriminating the depression of vulnerable elderly in urban area. J Korean Data Anal Soc, 2010, 12: 2541-2554.

10) Kim YK, Lee JW: Influencing factors on life satisfaction in female elderly with degenerative knee arthritis-pain. activity of daily living (ADL) depression. J Korean Data Anal Soc, 2011, 13: 3009-3020.

11) Wilson K, Mottram P, Sixsmith A, et al.: Depression symptoms in the very old living alone: prevalence, incidence and risk factors. Int J Geriatr Psychiatry, 2007, 22: 361-366. [Medline] [CrossRef]

12) Crane PB: Fatigue and physical activity in older women after myocardial infarction. Heart Lung, 2005, 34: 30-38. [Medline] [CrossRef]

13) Yoo IY, Lim MK, Yoo WS, et al.: A study on depression, self-reported health status and social support in elderly people. J Korean Gerontol Nurs, 2002, 4: 153-162.

14) Bak SS, Kim KS: A study about depression of elderly women living alone. J Korean Data Anal Soc, 2010, 12: 2555-2566.

15) Kim JH, Kim KB: Comparative study of perceived health status, self-esteem, depression, and life satisfaction between institutionalized elders and elders living home. J Korean Gerontol Nurs, 2008, 10: 182-192.

16) Yoo JY, Im BH: A case study of major disease distributions and patterns of depression of the elderly inpatients of geriatric hospital. J KAHP, 2007, 21: 117-135.

17) Seo SH, Rhee KH: The effect of residential relocation for the life of the elderly. J Korean Geriatr Soc, 1996, 16: 69-82.

18) Kang YA: Normative study of the Korean-mini state examination (KMMSE) in the elderly. Korean J Psychol, 2006, 25: 1-12.

19) Sheikh JI, Yesavage JA: Geriatric depression scale (GDS): Recent evidence and development of a shorter version. Clinical Gerontology : A Guide to Assessment and Intervention. New York: The Haworth Press, 1986, pp 165-173.

20) Ki B: SA preliminary study for the standardization of geriatric depression scale short form-Korea version. J Korean Neuropsychiatr Assoc, 1996, 35: 298-307.

21) Song MS: Construction of a functional status prediction model for the elderly. Doctoral Thesis, Seoul National University, 1991.

22) Rosenberg M: Society and adolescent self image. Princeton: Pricetion University Press, 1965.

23) Jang SA: A study about the effect of social support on the satisfaction with 
of the elderly institutionalized people. Seoul National University Master's Thesis, 1997.

24) Park JW: A study to development a scale of social support. Yonsei University Doctoral Thesis, 1985.

25) Kim M, Kweon YR: Predictors of depression in residents of geriatric medical and welfare facilities. J Korean Acad Psychiatr Ment Health Nurs, 2010, 19: 212-219.

26) Shin DS: BMI, depression and fluid deficit in hospitalized elders. Korean J Fundam Nurs, 2007, 14: 83-91.
27) Lee MJ, Rhee KO: Relationship between impaired elders satisfaction with care and their depression. J Korean Geriatr Soc, 2005, 25: 119-131.

28) Seo HS, Jung SD, Kim CS, et al.: Comparison of the depression and anxiety between the elderly in the home for the aged and those in the community. Yeungnam Univ J Med, 1992, 9: 256-268.

29) Kang JS, Chung YS: The influences of physical health, cognitive symptom and nutritionl status on the depression of the elderly dwelling in a big city. J Korean Acad Community Health Nurs, 2008, 19: 378-387. 